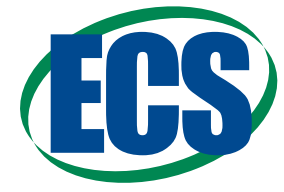

JeS Focus Issue on Mathematical. Modeling of Electrochemical Systems at Multiple Scales in Honor of John Newman

\title{
The Influence of Homogeneous Reactions on the Impedance Response of a Rotating Disk Electrode
}

\author{
Morgan S. Harding, ${ }^{\mathrm{a}, *}$ Bernard Tribollet, ${ }^{\mathrm{b}, * *}$ Vincent Vivier, $^{\mathrm{b}, * * *}$ and Mark E. Orazem ${ }^{\mathrm{a}, * *, \mathrm{z}}$ \\ ${ }^{a}$ Department of Chemical Engineering, University of Florida, Gainesville, Florida 32611, USA \\ ${ }^{b}$ UPR15 du CNRS, Laboratoire Interfaces et Systèmes Electrochimiques, Université P. et M. Curie, 75252 Paris, France
}

\begin{abstract}
A mathematical model was developed for the impedance response associated with coupled homogeneous chemical and heterogeneous electrochemical reactions. The model includes a homogeneous reaction in the electrolyte in which species $\mathrm{AB}$ reacts reversibly to form $\mathrm{A}^{-}$and $\mathrm{B}^{+}$and $\mathrm{B}^{+}$reacts electrochemically on a rotating disk electrode to produce $\mathrm{B}$. The resulting diffusion impedance has two asymmetric capacitive loops, one associated with convective diffusion and the other with the homogeneous reaction. For an infinitely fast homogeneous reaction, the system is shown to behave as though $\mathrm{AB}$ is the electroactive species. A modified Gerischer impedance was found to provide a good fit to the simulated data.

(c) The Author(s) 2017. Published by ECS. This is an open access article distributed under the terms of the Creative Commons Attribution 4.0 License (CC BY, http://creativecommons.org/licenses/by/4.0/), which permits unrestricted reuse of the work in any medium, provided the original work is properly cited. [DOI: 10.1149/2.0411711jes] All rights reserved.

(cc) BY
\end{abstract}

Manuscript submitted April 6, 2017; revised manuscript received May 23, 2017. Published June 15, 2017. This paper is part of the JES Focus Issue on Mathematical Modeling of Electrochemical Systems at Multiple Scales in Honor of John Newman.

The coupling of homogeneous chemical reactions and heterogeneous electrochemical reactions has drawn substantial interest over the past 80 years. Koutecky and Levich ${ }^{1-3}$ developed a steady-state model for a homogeneous reaction coupled with an electrochemical reaction on a rotating disk electrode. The homogenous reaction was assumed to follow linear kinetics, all diffusion coefficients were assumed to be equal, and the Schmidt number was assumed infinite. Koutecky and Levich defined a characteristic dimension for the homogeneous reaction, termed the thickness of the kinetic layer and represented by

$$
\delta_{\mathrm{r}}=\sqrt{\frac{D}{\alpha}}
$$

where $\alpha=k_{\mathrm{f}}+k_{\mathrm{b}}, k_{\mathrm{f}}$ and $k_{\mathrm{b}}$ are respectively the forward and backward rate constants of the homogeneous reaction, and $D$ is the diffusion coefficient.

Bossche et al. $^{4}$ describe finite-difference calculations under assumption of a steady state for an electrochemical system controlled by diffusion, migration, convection, and nonlinear homogeneous reaction kinetics. Their convection term used a three-term expansion appropriate for positions close to the electrode surface. ${ }^{5}$ Deslouis et al. ${ }^{6}$ used a submerged impinging jet cell to measure interfacial $\mathrm{pH}$ during the reduction of dissolved oxygen in the presence of carbonate. Their analysis considered the homogeneous reaction involving water and hydroxide, bicarbonate, and carbonate ions. Remita et al. ${ }^{7}$ have shown that, for a deaerated aqueous electrolyte containing dissolved carbon dioxide, hydrogen evolution is enhanced by the homogeneous dissociation of $\mathrm{CO}_{2}$. Tran et al. ${ }^{8}$ demonstrated that homogeneous dissociation of acetic acid enhances cathodic reduction of hydronium ions.

Smith ${ }^{9,10}$ used AC Polarography to study different linear firstorder homogeneous reaction mechanisms, including preceding, following, and catalytic chemical reactions coupled with electrochemical reactions. Jurczakowski and Polczynski ${ }^{11}$ developed an AC model with coupled homogenous and heterogeneous reactions accounting for cases where diffusion coefficients are not considered to be equal. The above mechanisms assumed simplified homogeneous reactions, with a maximum of two species considered.

Using chronopotentiometry, Delahay and Berzins ${ }^{12}$ showed that cadmium cyanide complexes undergo a dissociation before electrore-

\footnotetext{
*Electrochemical Society Student Member

***Electrochemical Society Fellow.

***Electrochemical Society Member.

zE-mail: meo@che.u.edu
}

duction on a mercury electrode. Savéant and Vianello ${ }^{13}$ devised the theoretical approach of the EC mechanism in the case of cyclic voltametry and proposed a kinetic zone diagram representing the various regimes of competition. From the theoretical development of polarography at a stationary electrode, Nicholson and Shain ${ }^{14}$ developed diagnostic criteria for following, preceding, and catalytic chemical reactions coupled with charge transfer.

In 1951, Gerischer ${ }^{15}$ published the first formal treatment of coupled chemical and electrochemical reactions under steady-state and oscillating steady-state (impedance) conditions. He considered a linear homogeneous reaction, equal diffusion coefficients, and a Nernst stagnant diffusion layer. A summary of Gerischer's derivation is presented by Lasia. ${ }^{16}$ Recently, Pototskayaa and Gichan ${ }^{17}$ extended the Gerischer impedance to account for a roughened electrode and nonidentical diffusion coefficients. Following Gerischer, Pototskayaa and Gichan invoked linear homogeneous kinetics and a Nernst stagnant diffusion layer. Chapman and Antaño ${ }^{18}$ discussed the use of orthogonal collocation as a means to explore the influence of nonlinear homogeneous reactions on the impedance response within a Nernst stagnant diffusion layer.

Levart and Schuhmann ${ }^{19}$ developed a model for the convective diffusion impedance of a disk electrode under assumption of a finite Schmidt number in the presence of a homogeneous chemical reaction. The diffusion coefficients of the substances involved in the reaction were assumed equal, and a linear expression for the homogeneous reaction was assumed. Tribollet and Newman ${ }^{20}$ described a model for concentrated solutions, based on the Stefan-Maxwell equations, with provision for an arbitrary number of simultaneous homogeneous and heterogeneous reactions. Their model was employed by Hauser and Newman ${ }^{21,22}$ to describe the influence of homogenous consumption of cuprous ion on the impedance response associated with dissolution of a rotating copper disk electrode under assumption of linear homogeneous kinetics and an infinite Schmidt number. Vazquez-Arenas and Pritzker $^{23,24}$ developed a model for the deposition of cobalt ions on a rotating cobalt disk under the assumption that homogeneous reactions were equilibrated.

A Gerischer-type impedance has been used to fit many electrode processes, including solid oxide fuel cell systems, ${ }^{25-27}$ oxide electrode systems, ${ }^{28}$ mixed conducting solid electrolyte systems, ${ }^{29}$ systems with boundary conditions on a disordered boundary ${ }^{30}$ and electrocatalytic systems influenced by the hydrogen evolution reaction. ${ }^{31}$ Coupled electrochemical and enzymatic homogeneous reactions are also involved in sensors used to monitor glucose concentrations for management of diabetes. ${ }^{32-34}$

The object of the present work was to relax the assumptions implicit in previously published papers by developing a model for the 


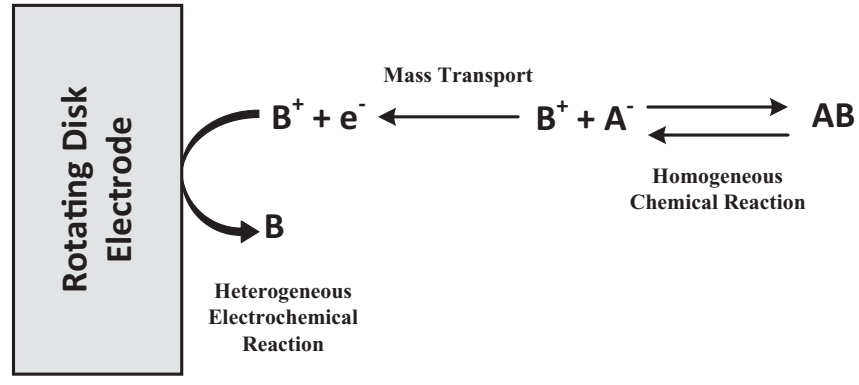

Figure 1. Schematic representation of an electrochemical reaction coupled by the influence of a homogeneous chemical reaction.

convective-diffusion impedance response of a rotating disk electrode for a finite Schmidt number, un-equal diffusion coefficients, and using nonlinear expressions for the kinetics of homogeneous reactions. The resulting impedance responses were compared to a hybrid Gerischer impedance that accounts for linear homogeneous reaction kinetics, equal diffusion coefficients, and a finite Schmidt number for the convective diffusion part of the expression.

\section{Mathematical Development}

A mathematical model is presented for the impedance response associated with the coupling of homogeneous and heterogeneous electrochemical reactions.

Governing equations.-The flux density of species i in a dilute electrolyte and in absence of migration was expressed as

$$
\mathbf{N}_{\mathrm{i}}=-D_{\mathrm{i}} \nabla c_{\mathrm{i}}+c_{\mathrm{i}} \mathbf{v}
$$

where $D_{\mathrm{i}}$ is the diffusion coefficient, $c_{\mathrm{i}}$ is the concentration of species $\mathrm{i}$, and $\mathbf{v}$ is the mass-averaged velocity. ${ }^{35}$ For an axisymmetric rotatingdisk electrode, the convective-diffusion equation with homogeneous reaction was expressed in cylindrical coordinates as

$$
\frac{\partial c_{\mathrm{i}}}{\partial t}+v_{\mathrm{y}} \frac{\partial c_{\mathrm{i}}}{\partial y}=D_{\mathrm{i}} \frac{\partial^{2} c_{\mathrm{i}}}{\partial y^{2}}+R_{\mathrm{i}}
$$

where $R_{i}$ is the rate of production of species $i$ by homogeneous reactions, $y$ is the coordinate normal to the electrode surface, and $v_{\mathrm{y}}$ is the velocity in the axial direction.

Homogeneous reaction.-A schematic representation of an electrochemical reaction coupled with a chemical reaction is shown in Figure 1

where the reactions may be expressed as

$$
\mathrm{AB} \underset{k_{\mathrm{b}}}{\stackrel{k_{\mathrm{f}}}{\rightleftarrows}} \mathrm{A}^{-}+\mathrm{B}^{+}
$$

where $k_{\mathrm{f}}$ and $k_{\mathrm{b}}$ are rate constants for the homogeneous reaction. The species $\mathrm{B}^{+}$was assumed to be electroactive and consumed at the electrode following the irreversible reaction

$$
\mathrm{B}^{+}+\mathrm{e}^{-} \rightarrow \mathrm{B}
$$

The corresponding current density from the electrochemical reaction, which is dependent on concentration and potential, was expressed as

$$
i_{\mathrm{B}^{+}}=-K_{\mathrm{B}^{+}} c_{\mathrm{B}^{+}}(0) \exp \left(-b_{\mathrm{B}^{+}} V\right)
$$

where $K_{\mathrm{B}^{+}}$is the rate constant, $b_{\mathrm{B}^{+}}$is the transfer coefficient, $c_{\mathrm{B}^{+}}(0)$ is the concentration of $\mathrm{B}^{+}$at the electrode surface and $V$ is the applied potential referenced to an electrode located just outside the double layer. As the electroactive species is consumed, a concentration gradient of $\mathrm{B}^{+}$must exist near the electrode surface.

The reaction sequence given as Equations 4 and 5 is general and may be applied to neutral species as well as to charged species. This sequence could apply, for example, to dissociation of acetic acid to produce $\mathrm{H}^{+}$ions, which participate in a cathodic electrochemical reaction.

Far from the electrode surface, the species $\mathrm{AB}, \mathrm{A}^{-}$, and $\mathrm{B}^{+}$are assumed to be equilibrated; thus,

$$
K_{\mathrm{eq}}=\frac{k_{\mathrm{f}}}{k_{\mathrm{b}}}=\frac{c_{\mathrm{A}^{-}}(\infty) c_{\mathrm{B}^{+}}(\infty)}{c_{\mathrm{AB}}(\infty)}
$$

The reaction term was expressed as

$$
R_{\mathrm{A}^{-}}=R_{\mathrm{B}^{+}}=-R_{\mathrm{AB}}=k_{\mathrm{f}} c_{\mathrm{AB}}(y)-k_{\mathrm{b}} c_{\mathrm{A}^{-}}(y) c_{\mathrm{B}^{+}}(y)
$$

where $k_{\mathrm{f}}$ has units of inverse time and $k_{\mathrm{b}}$ has units of inverse concentration per time, i.e., $\mathrm{cm}^{3} / \mathrm{mol} \mathrm{s}$. Combination of Equations 7 and 8 yields

$$
R_{\mathrm{A}^{-}}=R_{\mathrm{B}^{+}}=-R_{\mathrm{AB}}=k_{\mathrm{b}}\left(K_{\mathrm{eq}} c_{\mathrm{AB}}(y)-c_{\mathrm{A}^{-}}(y) c_{\mathrm{B}^{+}}(y)\right)
$$

In Equation 9, $k_{\mathrm{b}}$ was independently explored and $K_{\mathrm{eq}}$ was assumed to be constant and calculated from bulk concentrations. The form of Equation 9 makes the steady-state problem nonlinear.

Velocity.-The steady flow created by an infinite disk rotating at a constant angular velocity in a fluid with uniform physical properties was first studied by von Kármán..$^{5}$ The axial velocity is represented by

$$
v_{\mathrm{y}}=\sqrt{\nu \Omega} H(\zeta)
$$

where $\nu$ is the kinematic viscosity, $\Omega$ is the rotation speed, and $H(\zeta)$ is a dimensionless function of dimensionless position, $\zeta=y \sqrt{\Omega / \nu}$.

The Navier-Stokes equations can be solved numerically when equations for the dimensionless radial, angular and axial velocities are inserted. As shown by Cochran, ${ }^{36} H(\zeta)$ can be written as two sets of series expansions, one close to the electrode, $H_{0}$, and one far from the electrode, $H_{\infty}$. For small values of $\zeta$ the expansion is

$$
H_{0}=-\mathrm{a} \zeta^{2}+\frac{1}{3} \zeta^{3}-\frac{\mathrm{b}}{6} \zeta^{4}+\ldots
$$

where $\mathrm{a}=0.5102326189$ and $\mathrm{b}=-0.6159220144 .{ }^{19}$ Numerical simulation of the convective-diffusion equation for systems involving homogeneous reaction requires a bounded velocity at $\zeta \rightarrow \infty$, a condition that is not satisfied by Equation 11. Far from the electrode, when $\zeta$ is large, the expansion equation for $H$ becomes

$$
\begin{aligned}
H_{\infty} & =-\alpha+\frac{2 \mathrm{~A}}{\alpha} \exp (-\alpha \zeta)-\frac{\mathrm{A}^{2}+\mathrm{B}^{2}}{2 \alpha^{3}} \exp (-2 \alpha \zeta) \\
& -\frac{\mathrm{A}\left(\mathrm{A}^{2}+\mathrm{B}^{2}\right)}{6 \alpha^{5}} \exp (-3 \alpha \zeta)+\ldots
\end{aligned}
$$

where $\alpha=0.88447441, \mathrm{~A}=0.93486353$, and $\mathrm{B}=1.2021175 .{ }^{37}$

To solve the conservation equations in the presence of homogeneous reactions, a velocity profile is required to describe the fluid flow for a larger domain than is described by Equations 10 and 11 . An interpolation formula was used to provide an analytic expression for velocity in terms of velocity expansions near the electrode and far from the electrode, i.e., ${ }^{38}$

$$
H=(1-f) H_{0}+f H_{\infty}
$$

where $H_{0}$ and $H_{\infty}$ are given by Equations 11 and 12, respectively. The interpolation function was defined as

$$
f=\frac{1}{1+\mathrm{e}^{-\alpha\left(\zeta-\zeta_{0}\right)}}
$$

where $\zeta_{0}$ was assigned a value of unity and $\alpha=25$ influences the sharpness of the transition from $v_{\mathrm{y}, 0}$ to $v_{\mathrm{y}, \infty}$. Equation 14 is similar to the Fermi-Dirac function applied in quantum mechanics to describe the distribution of fermions.

The axial components of the dimensionless velocity as a function of dimensionless distance from the electrode surface are shown in Figure 2. Equation 13 satisfies the velocities for small and large values of $\zeta$. The slight discontinuity in the velocity expression occurs in a region outside the Nernst diffusion layer thickness of $13.8 \mu \mathrm{m}$ (shown in 


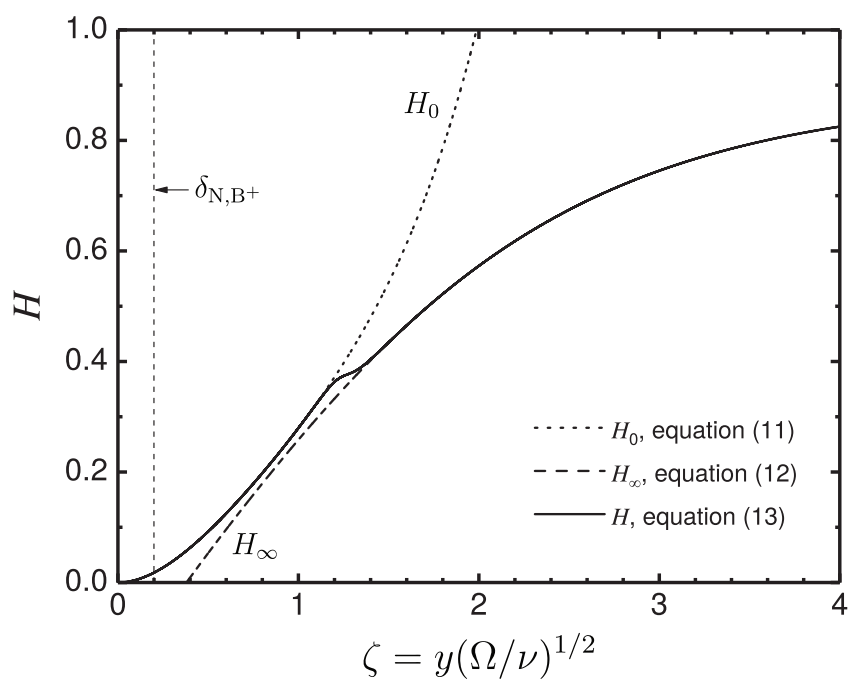

Figure 2. Dimensionless axial velocity expansions $H_{0}$, given as Equation 11, and $H_{\infty}$, given as Equation 12, and the interpolation function with $\alpha=25$ and $\zeta_{0}=1$, defined in Equation 14. The Nernst diffusion-layer thickness of 13.8 $\mu \mathrm{m}$ for a rotating disk electrode $(\mathrm{Sc}=526$ and $\Omega=2000 \mathrm{rpm}$ ) is illustrated as a vertical dashed line.

Figure 2 by a dashed line for $\mathrm{Sc}=526$ and $\Omega=2000 \mathrm{rpm}$ ) and, therefore, did not affect the simulation.

Impedance with homogeneous chemical reactions.-The conservation equation for each species was written as

$$
\frac{\partial c_{\mathrm{AB}}}{\partial t}+v_{\mathrm{y}} \frac{\partial c_{\mathrm{AB}}}{\partial y}=D_{\mathrm{AB}} \frac{\partial^{2} c_{\mathrm{AB}}}{\partial \mathrm{y}^{2}}-k_{\mathrm{b}}\left(K_{\mathrm{eq}} c_{\mathrm{AB}}(y)-c_{\mathrm{A}^{-}}(y) c_{\mathrm{B}^{+}}(y)\right)
$$

for $\mathrm{AB}$,

$$
\frac{\partial c_{\mathrm{A}^{-}}}{\partial t}+v_{\mathrm{y}} \frac{\partial c_{\mathrm{A}^{-}}}{\partial y}=D_{\mathrm{A}^{-}} \frac{\partial^{2} c_{\mathrm{A}^{-}}}{\partial \mathrm{y}^{2}}+k_{\mathrm{b}}\left(K_{\mathrm{eq}} c_{\mathrm{AB}}(y)-c_{\mathrm{A}^{-}}(y) c_{\mathrm{B}^{+}}(y)\right)
$$

for $\mathrm{A}^{-}$, and

$$
\frac{\partial c_{\mathrm{B}^{+}}}{\partial t}+v_{\mathrm{y}} \frac{\partial c_{\mathrm{B}^{+}}}{\partial y}=D_{\mathrm{B}^{+}} \frac{\partial^{2} c_{\mathrm{B}^{+}}}{\partial \mathrm{y}^{2}}+k_{\mathrm{b}}\left(K_{\mathrm{eq}} c_{\mathrm{AB}}(y)-c_{\mathrm{A}^{-}}(y) c_{\mathrm{B}^{+}}(y)\right)
$$

for $\mathrm{B}^{+}$. The boundary conditions far from the electrode were

$$
c_{\mathrm{i}} \rightarrow c_{\mathrm{i}}(\infty) \text { for } y \rightarrow \infty
$$

and the boundary conditions at the electrode surface were

$$
\left.\frac{\partial c_{\mathrm{i}}}{\partial \mathrm{y}}\right|_{y=0}=0 \text { for } y=0
$$

for the non-reacting species, and

$$
\left.\mathrm{F} D_{\mathrm{B}^{+}} \frac{\partial c_{\mathrm{B}^{+}}}{\partial \mathrm{y}}\right|_{y=0}=i_{\mathrm{B}^{+}} \text {for } y=0
$$

for the reacting species $\mathrm{B}^{+}$. The concentrations of each species were represented in terms of steady-state and oscillating terms as ${ }^{39,40}$

$$
c_{\mathrm{i}}=\bar{c}_{\mathrm{i}}+\operatorname{Re}\left\{\widetilde{c}_{\mathrm{i}} \exp (\mathrm{j} \omega t)\right\}
$$

The resulting equations governing the steady-state were

$$
\begin{aligned}
& v_{\mathrm{y}} \frac{\partial \bar{c}_{\mathrm{AB}}}{\partial y}=D_{\mathrm{AB}} \frac{\partial^{2} \bar{c}_{\mathrm{AB}}}{\partial \mathrm{y}^{2}}-\bar{R}_{\mathrm{AB}} \\
& v_{\mathrm{y}} \frac{\partial \bar{c}_{\mathrm{A}^{-}}}{\partial y}=D_{\mathrm{A}^{-}} \frac{\partial^{2} \bar{c}_{\mathrm{A}^{-}}}{\partial \mathrm{y}^{2}}+\bar{R}_{\mathrm{A}^{-}}
\end{aligned}
$$

and

$$
v_{\mathrm{y}} \frac{\partial \bar{c}_{\mathrm{B}^{+}}}{\partial y}=D_{\mathrm{B}^{+}} \frac{\partial^{2} \bar{c}_{\mathrm{B}^{+}}}{\partial \mathrm{y}^{2}}+\bar{R}_{\mathrm{B}^{+}}
$$

where

$$
\bar{R}_{\mathrm{A}^{-}}=\bar{R}_{\mathrm{B}^{+}}=-\bar{R}_{\mathrm{AB}}=k_{\mathrm{b}}\left(K_{\mathrm{eq}} \bar{c}_{\mathrm{AB}}(y)-\bar{c}_{\mathrm{A}^{-}}(y) \bar{c}_{\mathrm{B}^{+}}(y)\right)
$$

The equations governing the frequency domain were

$$
\begin{aligned}
& \mathrm{j} \omega \widetilde{c}_{\mathrm{AB}}+v_{\mathrm{y}} \frac{\partial \widetilde{c}_{\mathrm{AB}}}{\partial y}=D_{\mathrm{AB}} \frac{\partial^{2} \widetilde{c}_{\mathrm{AB}}}{\partial \mathrm{y}^{2}}-\widetilde{R}_{\mathrm{AB}} \\
& \mathrm{j} \omega \widetilde{c}_{\mathrm{A}^{-}}+v_{\mathrm{y}} \frac{\partial \widetilde{c}_{\mathrm{A}^{-}}}{\partial y}=D_{\mathrm{A}^{-}} \frac{\partial^{2} \widetilde{c}_{\mathrm{A}^{-}}}{\partial \mathrm{y}^{2}}+\widetilde{R}_{\mathrm{A}^{-}}
\end{aligned}
$$

and

$$
\mathrm{j} \omega \widetilde{c}_{\mathrm{B}^{+}}+v_{\mathrm{y}} \frac{\partial \widetilde{c}_{\mathrm{B}^{+}}}{\partial y}=D_{\mathrm{B}^{+}} \frac{\partial^{2} \widetilde{c}_{\mathrm{B}^{+}}}{\partial \mathrm{y}^{2}}+\widetilde{R}_{\mathrm{B}^{+}}
$$

where

$$
\begin{aligned}
\widetilde{R}_{\mathrm{A}^{-}} & =\widetilde{R}_{\mathrm{B}^{+}}=-\widetilde{R}_{\mathrm{AB}} \\
& =k_{\mathrm{b}}\left[K_{\mathrm{eq}} \widetilde{c}_{\mathrm{AB}}(y)-\bar{c}_{\mathrm{A}^{-}}(y) \widetilde{c}_{\mathrm{B}^{+}}(y)-\widetilde{c}_{\mathrm{A}^{-}}(y) \bar{c}_{\mathrm{B}^{+}}(y)\right]
\end{aligned}
$$

Following the usual hypothesis for impedance, the system response to a small perturbation amplitude behaves linearly, and thus the terms $\mathcal{O}\left(\tilde{c}^{2}\right)$ and greater have been neglected. Equations 26-29 are complex, coupled, and linear and are dependent on the steady-state solution. The boundary conditions for the oscillating concentrations were

$$
\tilde{c}_{\mathrm{i}}=0 \text { for } y \rightarrow \infty
$$

for each species

$$
\left.\frac{\partial \widetilde{c}_{\mathrm{i}}}{\partial \mathrm{y}}\right|_{y=0}=0 \text { for } y=0
$$

for the $\mathrm{AB}$ and $\mathrm{A}^{-}$, and

$$
\widetilde{c}_{\mathrm{B}^{+}}(0)=1 \text { for } \quad y=0
$$

for the reacting species $\mathrm{B}^{+}$. The value of $\widetilde{c}_{\mathrm{B}^{+}}(0)$ was chosen arbitrarily because the governing equations for the impedance response are linear, even when the steady-state problem is nonlinear. Application of the nonlinear homogeneous reaction kinetics to impedance response represents an extension to the literature.

Diffusion impedance.-The oscillating current density associated with $\mathrm{B}^{+}$was expressed as a Taylor series expansion about the steadystate current, shown in Equation 6, as ${ }^{39,40}$

$$
\widetilde{i}_{\mathrm{B}^{+}}=\left(\frac{\partial i_{\mathrm{B}^{+}}}{\partial V}\right)_{c_{\mathrm{B}^{+}}(0)} \widetilde{V}+\left(\frac{\partial i_{\mathrm{B}^{+}}}{\partial c_{\mathrm{B}^{+}}(0)}\right)_{V} \widetilde{c}_{\mathrm{B}^{+}}(0)
$$

where $\widetilde{V}$ and $\widetilde{c}_{\mathrm{B}^{+}}(0)$ are assumed to have a small magnitude such that the higher order terms can be neglected. The flux expression for $\mathrm{B}^{+}$in the absence of migration yields a second equation for the oscillating current density as

$$
\widetilde{i}_{\mathrm{B}^{+}}=\left.\mathrm{F} D_{\mathrm{B}^{+}} \frac{\mathrm{d} \widetilde{c}_{\mathrm{B}^{+}}}{\mathrm{d} y}\right|_{y=0}
$$

Equation 33 was divided by Equation 34, yielding

$$
1=\left(\frac{\partial i_{\mathrm{B}^{+}}}{\partial V}\right)_{c_{\mathrm{B}}+(0)} \frac{\widetilde{V}}{\tilde{i}_{\mathrm{B}^{+}}}+\left(\frac{\partial i_{\mathrm{B}^{+}}}{\partial c_{\mathrm{B}^{+}}(0)}\right)_{V} \frac{\widetilde{c}_{\mathrm{B}^{+}}(0)}{\left.\mathrm{F} D_{\mathrm{B}^{+}} \frac{\mathrm{d} \widetilde{c}_{\mathrm{B}^{+}}}{\mathrm{d} y}\right|_{y=0}}
$$

The faradaic contribution to the impedance is defined by

$$
Z_{\mathrm{F}, \mathrm{B}^{+}}(\omega)=\frac{\widetilde{V}}{\tilde{i}_{\mathrm{B}^{+}}}
$$




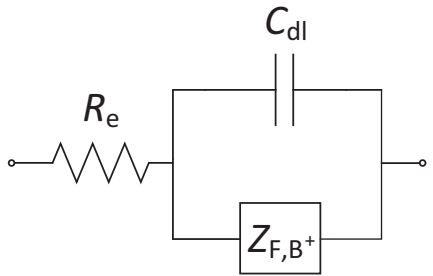

Figure 3. Electrical circuit representation of the electrode impedance associated with the faradaic impedance given as Equation 37.

where $\omega$ is the angular frequency. Thus, by rearranging Equation 35, the faradaic contribution to the impedance was expressed as

$$
Z_{\mathrm{F}, \mathrm{B}^{+}}(\omega)=R_{\mathrm{t}, \mathrm{B}^{+}}+Z_{\mathrm{D}, \mathrm{B}^{+}}(\omega)
$$

where, from the respective derivatives of Equation 6, the charge transfer resistance was given as

$$
R_{\mathrm{t}, \mathrm{B}^{+}}=\frac{1}{K_{\mathrm{B}^{+}} b_{\mathrm{B}^{+}} \bar{c}_{\mathrm{B}^{+}}(0) \exp \left(-b_{\mathrm{B}^{+}} \bar{V}\right)}
$$

and the diffusion impedance was given as

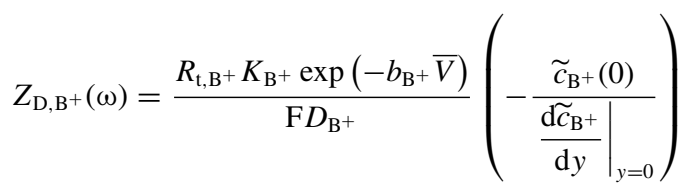

The concentration distributions required to assess the diffusion impedance, Equation 39, were obtained for each frequency from the numerical solution of Equations 26-29.

The dimensionless diffusion impedance is given by

$$
-\frac{1}{\theta_{\mathrm{B}^{+}}^{\prime}}(K)=\frac{1}{\delta_{\mathrm{N}, \mathrm{B}^{+}}}\left(-\frac{\widetilde{c}_{\mathrm{B}^{+}}(0)}{\left.\frac{\mathrm{d} \widetilde{c}_{\mathrm{B}^{+}}}{\mathrm{d} y}\right|_{y=0}}\right)
$$

where $\delta_{\mathrm{N}, \mathrm{B}^{+}}$is the diffusion-layer thickness using the Nernst hypothesis, shown as

$$
\delta_{\mathrm{N}, \mathrm{B}^{+}}=\Gamma(4 / 3)\left(\frac{3}{\mathrm{a}}\right)^{1 / 3} \frac{1}{\mathrm{Sc}_{\mathrm{B}^{+}}^{1 / 3}} \sqrt{\frac{\nu}{\Omega}}
$$

$\Gamma$ is the gamma function, and the $\mathrm{Schmidt}$ number is defined as $\mathrm{Sc}_{\mathrm{B}^{+}}=$ $\nu / D_{\mathrm{B}^{+}}$. Equation 40 is a function of a dimensionless frequency

$$
K=\frac{\omega \delta_{\mathrm{B}^{+}}}{D_{\mathrm{B}^{+}}}
$$

where

$$
\delta_{\mathrm{B}^{+}}=\left(\frac{3}{\mathrm{a}}\right)^{1 / 3} \frac{1}{\mathrm{Sc}_{\mathrm{B}^{+}}^{1 / 3}} \sqrt{\frac{\nu}{\Omega}}
$$

The variables $K$ and $\delta_{\mathrm{B}^{+}}$are used to place the convective-diffusion equation in dimensionless form for an infinite Schmidt number. ${ }^{39,40}$

Overall impedance.-The overall impedance can be represented by the circuit shown in Figure 3. An ohmic resistance is in series with the parallel contribution of the faradaic impedance, Equation 37, and double-layer capacitance, yielding a mathematical expression for the overall impedance as

$$
Z(\omega)=R_{\mathrm{e}}+\frac{Z_{\mathrm{F}, \mathrm{B}^{+}}}{1+\mathrm{j} \omega Z_{\mathrm{F}, \mathrm{B}^{+}} C_{\mathrm{dl}}}
$$

where $Z_{\mathrm{F}, \mathrm{B}^{+}}$was obtained from Equation 37 .
Table I. Species and associated parameter values for the system.

\begin{tabular}{cccc} 
Species & $c_{\mathrm{i}}(\infty), \mathrm{mol} / \mathrm{cm}^{3}$ & $z_{\mathrm{i}}$ & $D_{\mathrm{i}}, \mathrm{cm}^{2} / \mathrm{s}$ \\
\hline $\mathrm{AB}$ & 0.01 & 0 & $1.684 \times 10^{-5}$ \\
$\mathrm{~A}^{-}$ & 0.0001 & -1 & $1.957 \times 10^{-5}$ \\
$\mathrm{~B}^{+}$ & 0.0001 & 1 & $1.902 \times 10^{-5}$
\end{tabular}

Table II. System and kinetic parameter values for the system.

\begin{tabular}{lcc} 
Parameter & Value & Units \\
\hline Disk rotation rate, $\Omega$ & 2,000 & $\mathrm{rpm}$ \\
Kinematic viscosity, $\nu$ & 0.01 & $\mathrm{~cm}^{2} / \mathrm{s}$ \\
Homogeneous equilibrium constant, $K_{\text {eq }}$ & $10^{-6}$ & $\mathrm{~mol} / \mathrm{cm}^{3}$ \\
Homogeneous rate constant, $k_{\mathrm{b}}$, & $10^{7}$ & $\mathrm{~cm}^{3} / \mathrm{mol} \mathrm{s}^{-12}$ \\
Heterogeneous rate constant, $K_{\mathrm{B}^{+}}$ & $2 \times 10^{-12}$ & $\mathrm{~A} / \mathrm{cm}^{2}$ \\
Heterogeneous constant, $b_{\mathrm{B}^{+}}$ & 19.9 & $\mathrm{~V}^{-1}$
\end{tabular}

Numerical methods. - The steady-state concentrations of $\mathrm{AB}, \mathrm{A}^{-}$, and $\mathrm{B}^{+}$and the value of the homogeneous reaction rate were obtained for input values shown in Tables I and II. These steady-state values were used to obtain the frequency-domain concentration of the reacting species $\mathrm{B}^{+}$. All the equations were linearized, formulated in finite-difference form and solved numerically using Newman's BAND method coupled with Newton-Raphson iteration..$^{35}$ The boundary corresponding to $y \rightarrow \infty$ was chosen to be located at a position $20 \delta_{\mathrm{N}, \mathrm{B}^{+}}$. Equation 40 was used to obtain a dimensionless diffusion impedance, and Equation 39 was used to obtain a diffusion impedance.

To minimize round-off and finite difference errors, a small mesh size $(5 \mathrm{~nm})$ was employed near the electrode, in an inner region, and a large mesh size $(60 \mathrm{~nm})$ was used in the outer region dominated by convection. The transition between regions allowed solutions with accuracy on the order of the square of the mesh size. The steady-state concentrations so determined had greater than 8 significant digits and the real and imaginary parts of the impedance were computed with 6 significant digits.

\section{Results}

The polarization curve corresponding to the parameters given in Tables I and II is presented in Figure 4. The heterogeneous reaction rate increases as the potential becomes more negative, reaching a mass-transfer-limited plateau for potentials smaller than $-2.2 \mathrm{~V}$. The large magnitude of the mass-transfer-limited current density may be attributed to the production of $\mathrm{B}^{+}$by the homogeneous reaction.

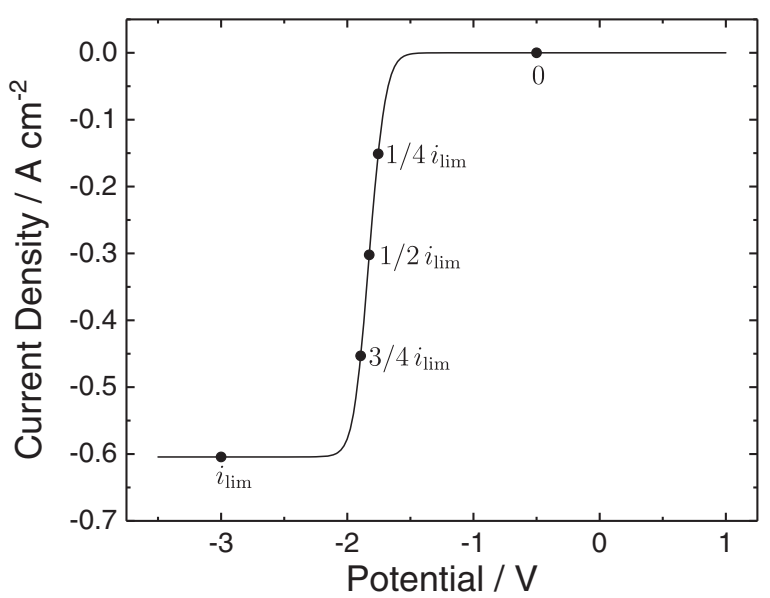

Figure 4. Polarization curve calculated for system parameters presented in Tables I and II. Labeled potential values at fractions of the limiting current correspond to steady-state concentration profiles presented in Figure 5. 


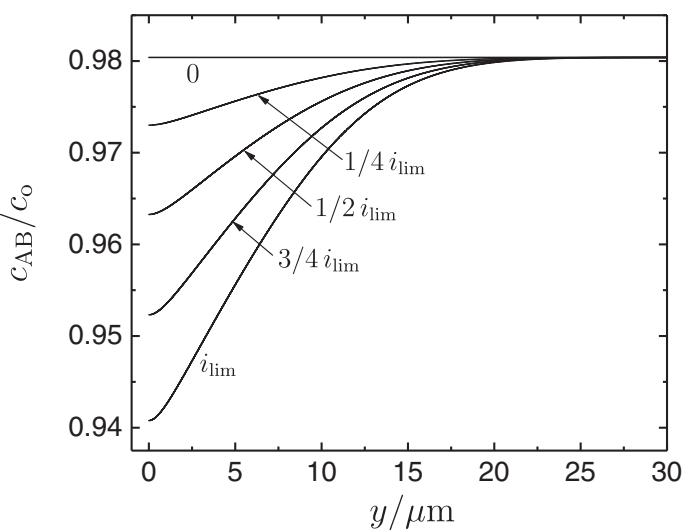

(a)

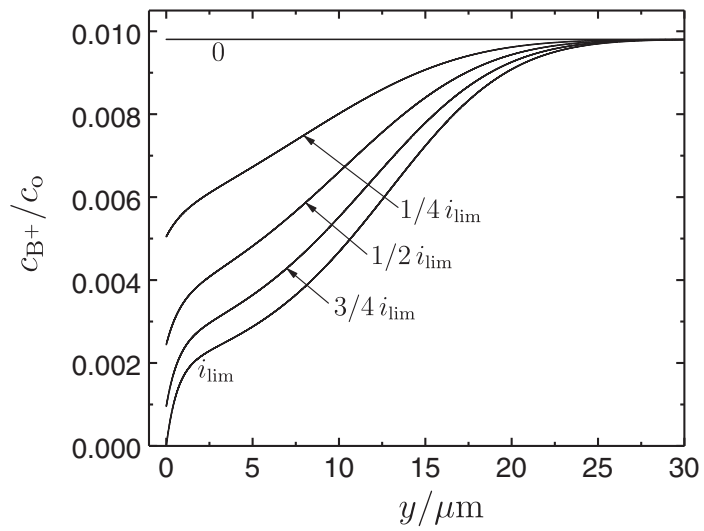

(c)

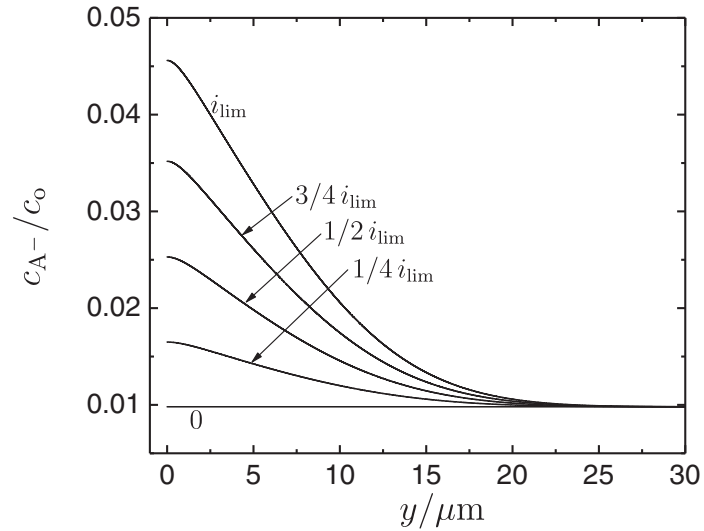

(b)

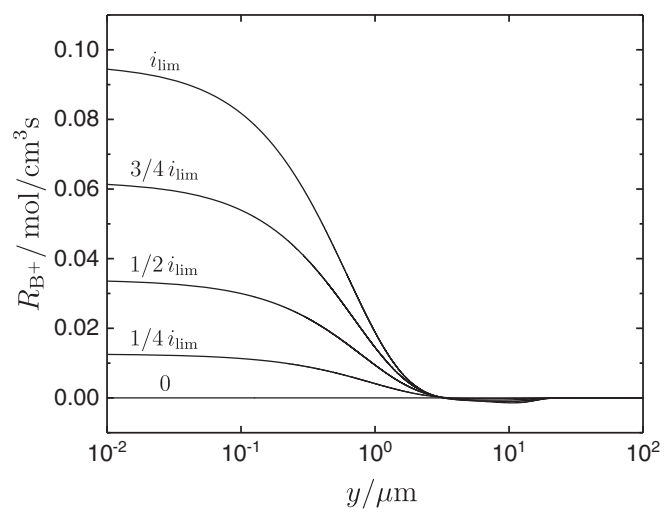

(d)

Figure 5. Calculated steady-state concentration distributions and homogeneous reaction rate corresponding to system parameters presented in Tables I and II at different fractions of the limiting current; a) $\mathrm{B}^{+}$b) $\mathrm{A}^{-}$c) $\mathrm{AB}$ d) $R_{\mathrm{B}^{+}}$.

Labeled fractions of the mass-transfer-limited current density values in Figure 4 correspond to steady-state concentration profiles and the reaction profile presented in Figure 5. Concentrations were scaled by the mass balance of the species involved in the homogeneous reaction, $c_{\mathrm{o}}=c_{\mathrm{A}^{-}}+c_{\mathrm{B}^{+}}+c_{\mathrm{AB}}$, to emphasize the relative changes in values as well as the overall concentration in the electrolyte. The concentration of AB, shown in Figure 5a, decreases to a value that is 94 percent of $c_{\mathrm{o}}$ at a potential corresponding to a value in the mass-transferlimited current range. In contrast, the concentration of $\mathrm{A}^{-}$shown in Figure $5 \mathrm{~b}$ reaches a value that is almost 5 times its bulk value at the

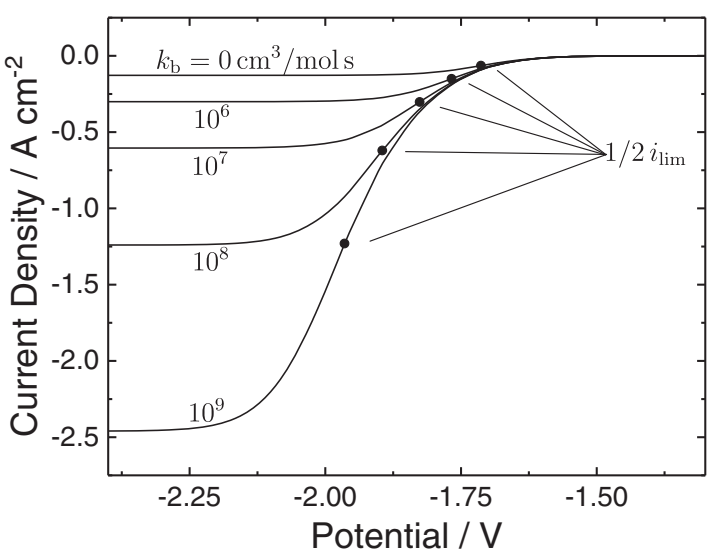

(a) mass-transfer-limited current density. The normalized concentration distribution of $\mathrm{B}^{+}$is presented in Figure $5 \mathrm{c}$. The concentration of $\mathrm{B}^{+}$at the electrode surface approaches a value of zero as the masstransfer-limited current density is approached. The sharp profile that appears close to the electrode surface in Figure $5 \mathrm{c}$ is consistent with a large current density. To emphasize the profile near the electrode, the rate of the homogeneous reaction is presented in Figure $5 d$ as a function of position on a logarithmic scale.

The influence of the homogeneous reaction rate constant on current density can be seen in Figure 6a, where a homogeneous rate

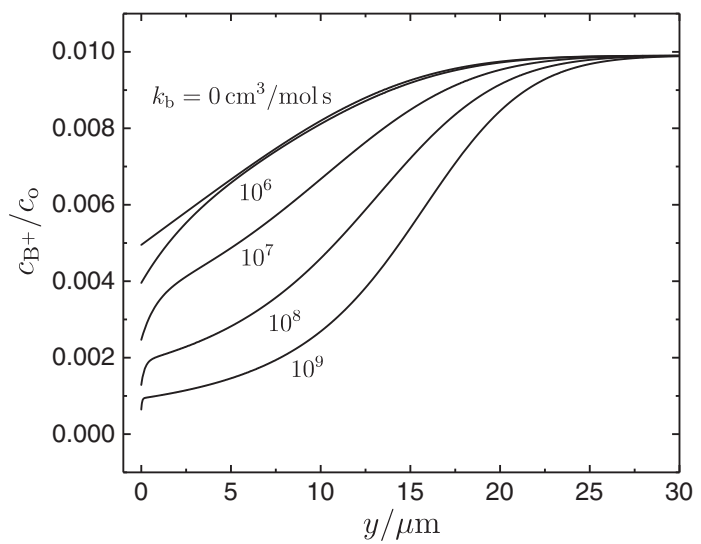

(b)

Figure 6. Calculations corresponding to system parameters presented in Tables I and II with homogeneous rate constant $k_{\mathrm{b}}$ as a parameter: a) polarization curve b) concentration distribution for $\mathrm{B}^{+}$at half the mass-transfer-limiting current. 


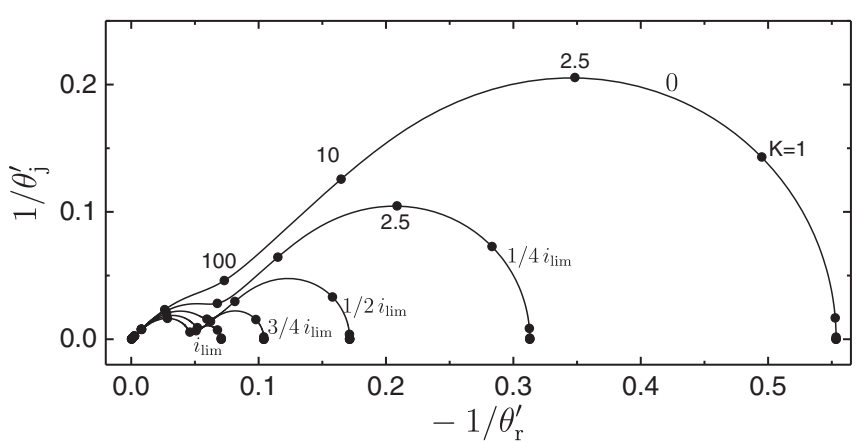

Figure 7. Dimensionless convective-diffusion impedance, defined by Equation 40, for the system presented in Figure 5 taken at fractions of the limiting current density.

constant of $k_{\mathrm{b}}=10^{9} \mathrm{~cm}^{3} / \mathrm{mol} \mathrm{s}$ yields a mass-transfer-limited current density that is almost 20 times larger than that in the absence of homogeneous reactions. The concentrations of $\mathrm{B}^{+}$corresponding to half the mass-transfer-limited current for different homogeneous reaction rate constants are presented in Figure $6 \mathrm{~b}$ as functions of position. In the absence of homogeneous reaction, the concentration profile is that expected for a rotating disk electrode. ${ }^{35}$ The slope at the electrodeelectrolyte interface becomes larger as the homogeneous rate constant increases.

The dimensionless convective-diffusion impedance corresponding to the steady-state results presented in Figure 5 are presented in Figure 7. For all potentials, two asymmetric capacitive loops are seen in Figure 7 as compared to a single loop in traditional convectivediffusion impedance. The low-frequency loop, for all cases, has a characteristic frequency, $K=2.5$, which is in agreement with the characteristic frequency associated with diffusion in the absence of homogeneous reactions. The low-frequency loop decreased in size as the potential corresponding to the mass-transfer-limited current density was approached. The high-frequency loop, present for all fractions of the mass-transfer-limited current density, corresponds to the homogeneous reaction and decreased slightly and became better defined at more cathodic potentials.

The individual impedance diagrams from Figure 7 are presented separately in Figure 8. Each dimensionless diffusion impedance corresponds to a point on the polarization curve, which is illustrated in Figure 8 by a line. The dimensionless-diffusion impedances are presented in a clockwise fashion, going from the most anodic potential to the most cathodic potential. The high-frequency loop is less defined

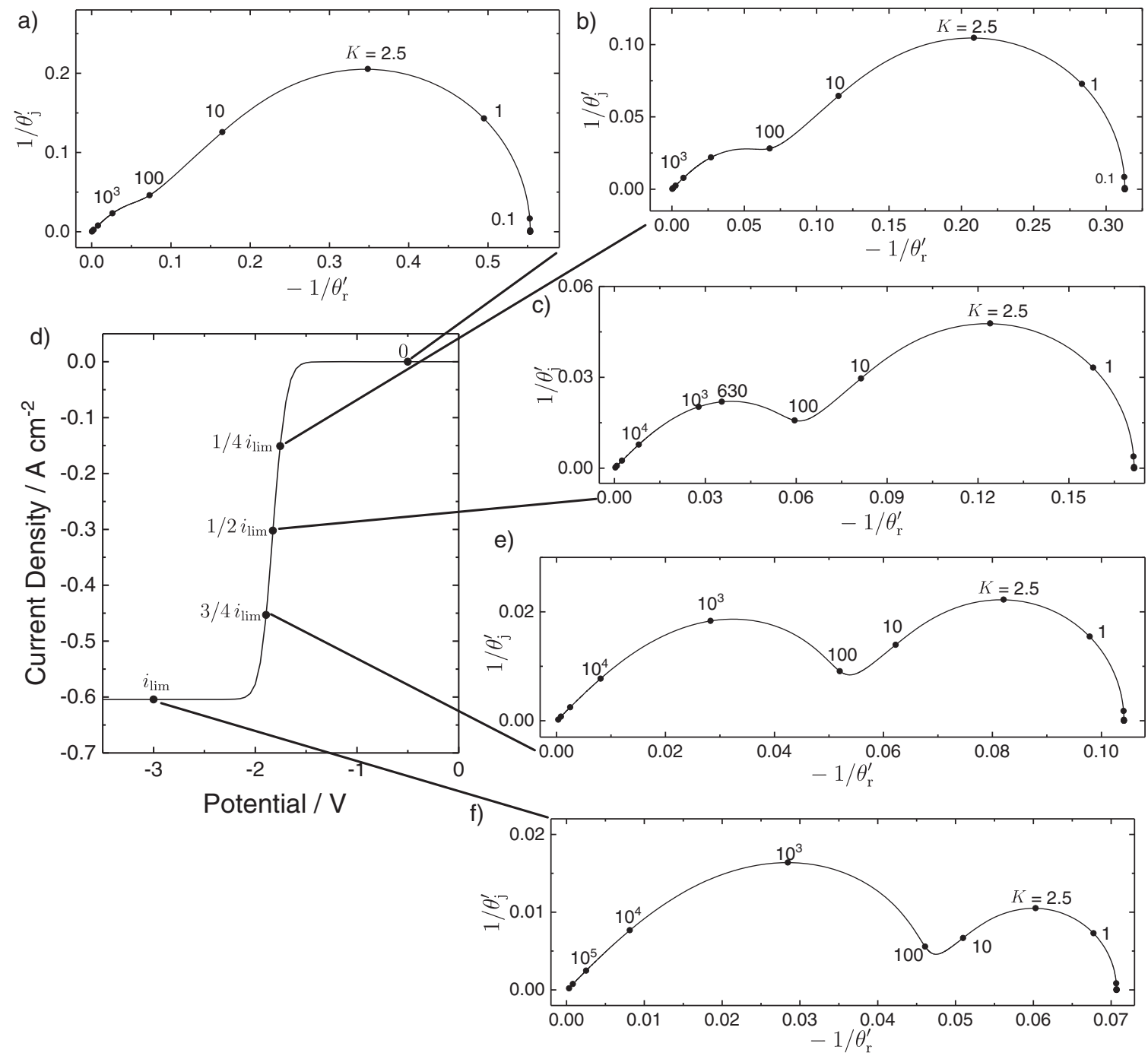

Figure 8. The dimensionless convective-diffusion impedance, defined by Equation 40 , corresponding to Figures 4 and 5 for different values of $i / i_{\text {lim }}$ : a) 0 ; b) $1 / 4$; c) $1 / 2$; e) $3 / 4$; and f) 1 . The dimensionless-diffusion impedances are presented in a clockwise fashion, going from the most anodic potential to the most cathodic potential. A line indicates the point on the polarization curve (d) corresponding to each Nyquist diagram. Dimensionless frequencies, $K=\omega \delta_{\mathrm{i}}^{2} / D_{\mathrm{i}}$, are labeled at characteristic frequencies as well as at relevant decade points. 


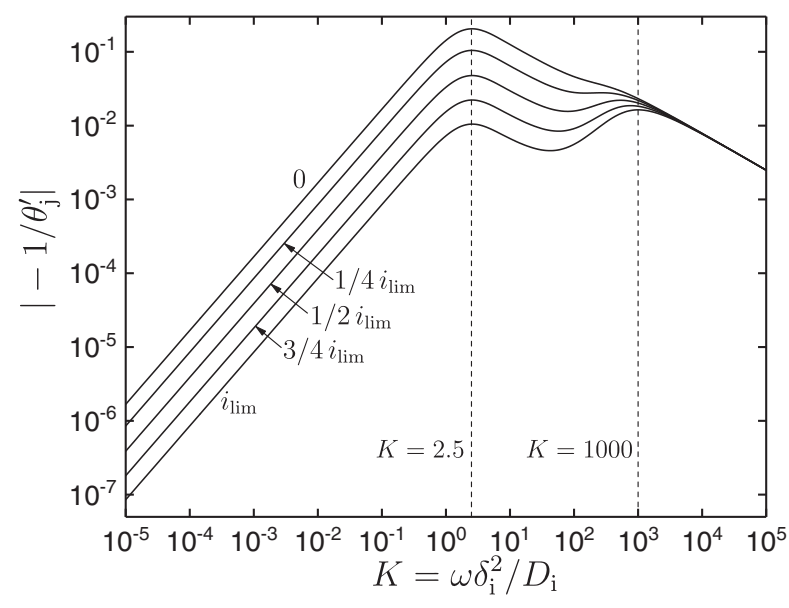

Figure 9. The absolute value of the imaginary part of the dimensionless convective-diffusion impedance, defined by Equation 40, for the system presented in Figure 5 as a function of potentials taken at fractions of the limiting current as a parameter. The characteristic frequency for convective-diffusion, at $K=2.5$, and the approximate characteristic frequency associated with the homogeneous reaction, at $K=1000$, are illustrated with dashed vertical lines.

for more anodic potentials, as shown in Figures 8a and 8b. At more negative potentials, the high-frequency loop resembles a Gerischer impedance element, as shown in Figures 8c, 8e and 8f. The characteristic frequency for the high-frequency loop is on the order of $K=1000$, suggesting that the characteristic dimension for the reaction is much smaller than the Nernst diffusion-layer thickness.

The two characteristic frequencies are better illustrated in Figure 9, where the absolute value of the dimensionless imaginary impedance is plotted as a function of dimensionless frequency. The characteristic frequency for convective-diffusion, at $K=2.5$, and the approximate characteristic frequency associated with the homogeneous reaction, at $K=1000$, are illustrated with dashed vertical lines in Figure 9.

The cell impedance was calculated following Equation 3 and Figure 3, under the assumptions that $R_{\mathrm{e}}=10 \Omega \mathrm{cm}^{2}, C_{\mathrm{dl}}=$ $20 \mu \mathrm{F} / \mathrm{cm}^{2}$, and the faradaic impedance was given by Equation 44 . The results are presented in Figure 10. Each impedance diagram is connected to the corresponding current density on the polarization curve by a line. The impedance diagrams are also presented in a clockwise fashion, from the most anodic potential to the most cathodic potential. The high-frequency loops presented in Figures 10b, 10d, and 10e decreased in size with increasing fraction of the limiting current density, indicating that the high frequency loop can be attributed to the charge-transfer resistance. The low-frequency loops have a characteristic frequency, $K=2.5$, that corresponds to convective-diffusion impedance. The loop at intermediate frequencies can be attributed to the homogenous reaction.

At potentials of $-0.5 \mathrm{~V}$ and $-3.0 \mathrm{~V}$, the impedance response appears almost capacitive as might be expected when the current is almost insensitive to applied potential, as shown in Figure 4. The expanded frequency range shown in Figure 11 indicated that the response in Nyquist format has some curvature, but this curvature is evident only at very low frequencies, generally considered to be unaccessible experimentally. At a potential of $-0.5 \mathrm{~V}$, the charge-transfer resistance is very large, as would be expected for an irreversible reaction. The potential of $-3.0 \mathrm{~V}$ represents the mass-transfer limit, where, as discussed in Tran et al., ${ }^{41}$ the charge-transfer resistance approaches a finite value, and the diffusion impedance becomes very large.

\section{Discussion}

Simulations of the steady-state concentrations and reaction distribution and convective-diffusion impedance for a rotating disk electrode influenced by homogenous reaction give insight to the impedance response of an electrochemical system influenced by ho- mogenous reaction. The diffusion impedances have two capacitive loops. The present section provides a development of kinetic and mass-transfer expressions for fast homogeneous reactions and the application of Gerischer impedance as a means to interpret experimental data conforming to the assumptions associated with the present mathematical model.

Fast homogeneous reaction.-In the limit of an infinitely fast homogeneous reaction, Reactions 4 and 5 can be expressed as

$$
\mathrm{AB}+\mathrm{e}^{-} \rightarrow \mathrm{A}^{-}+\mathrm{B}^{+}
$$

where $\mathrm{AB}$ is considered to be the electroactive species. The dimensionless diffusion impedance based on the oscillating concentration $\widetilde{c}_{\mathrm{AB}}$ is given by

$$
-\frac{1}{\theta_{\mathrm{AB}}^{\prime}}=\frac{1}{\delta_{\mathrm{N}, \mathrm{AB}}}\left(-\frac{\widetilde{c}_{\mathrm{AB}}(0)}{\left.\frac{\mathrm{d} \widetilde{c}_{\mathrm{AB}}}{\mathrm{d} y}\right|_{y=0}}\right)
$$

The convective-diffusion impedance given as Equation 46 is presented in Figure 12 with homogeneous rate constant as a parameter. For $k_{\mathrm{b}}=10^{8} \mathrm{~cm}^{3} / \mathrm{mol} \mathrm{s}$, the dimensionless diffusion impedance is very large and has the appearance of a distorted semi-circle. For $k_{\mathrm{b}}=10^{40} \mathrm{~cm}^{3} / \mathrm{mol} \mathrm{s}$, the dimensionless diffusion impedance takes the appearance of a usual dimensionless convective-diffusion impedance, with a characteristic frequency, based on the diffusion coefficient of species $\mathrm{AB}$, of $K=2.5$, a low-frequency limit of 1.0392 , and an angle with respect to the real axis of 45 degrees at the high-frequency limit. These results confirm that, for $k_{\mathrm{b}}=10^{40} \mathrm{~cm}^{3} / \mathrm{mol} \mathrm{s}$, Reactions 4 and 5 can be expressed as Reaction 45 .

Modified Gerischer impedance.-The convective-diffusion impedances presented in Figure 8 resembles a Gerischer impedance. The mathematical development for the Gerischer impedance is summarized in the following section. A modified Gerischer Impedance was regressed to simulation results to explore the manner in which simulatiuons may be employed to extract meaningful parameters, even if the assumptions implicit in the Gerischer model are not satisfied.

Mathematical description.-Under the assumptions that the diffusion coefficients for $\mathrm{AB}$ and $\mathrm{B}^{+}$are equal, that convection may be ignored, and that the concentration of $\mathrm{A}^{-}$is sufficiently large to be considered constant, Gerischer developed an analytic expression for the diffusion impedance associated with a heterogeneous reaction influenced by a homogeneous reaction in a Nernst stagnant diffusion layer. The rate of production of species $\mathrm{AB}$ and $\mathrm{B}^{+}$by Reaction 4 was expressed as

$$
R_{\mathrm{B}^{+}}=-R_{\mathrm{AB}}=k_{\mathrm{f}} c_{\mathrm{AB}}(y)-k_{\mathrm{b}} c_{\mathrm{B}^{+}}(y)
$$

The conservation equations for species $\mathrm{AB}$ and $\mathrm{B}^{+}$may be expressed as

$$
\frac{\partial c_{\mathrm{AB}}}{\partial t}=D \frac{\partial^{2} c_{\mathrm{AB}}}{\partial y^{2}}-k_{\mathrm{f}} c_{\mathrm{AB}}+k_{\mathrm{b}} c_{\mathrm{B}^{+}}
$$

and

$$
\frac{\partial c_{\mathrm{B}^{+}}}{\partial t}=D \frac{\partial^{2} c_{\mathrm{B}^{+}}}{\partial y^{2}}+k_{\mathrm{f}} c_{\mathrm{AB}}-k_{\mathrm{b}} c_{\mathrm{B}^{+}}
$$

respectively, where

$$
D=D_{\mathrm{AB}}=D_{\mathrm{B}^{+}}
$$

The sum of Equations 48 and 49 yields

$$
\frac{\partial}{\partial t}\left(c_{\mathrm{AB}}+c_{\mathrm{B}^{+}}\right)=D \frac{\partial^{2}}{\partial y^{2}}\left(c_{\mathrm{AB}}+c_{\mathrm{B}^{+}}\right)
$$



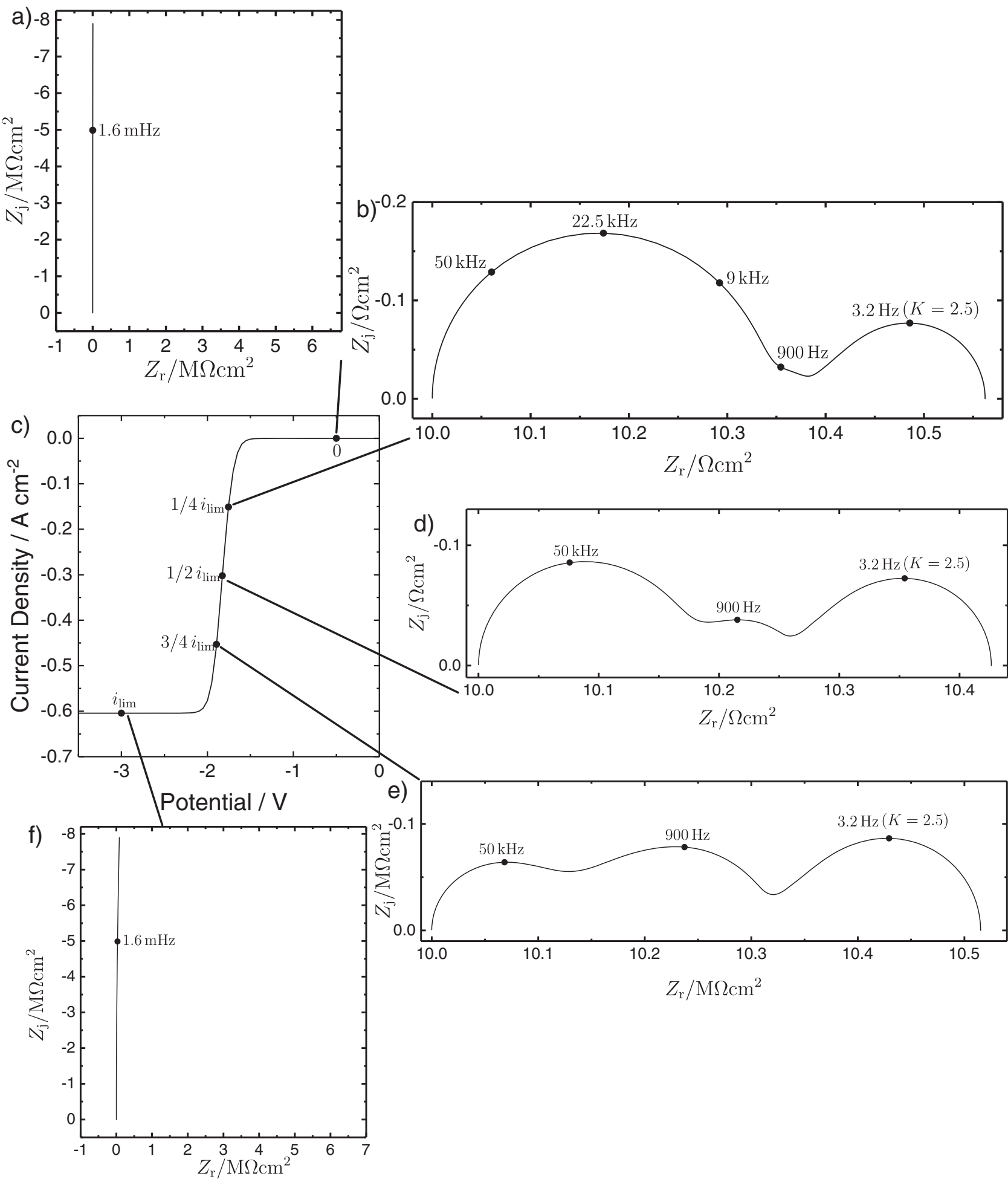

Figure 10. The overall impedance corresponding to Figure 8 with an ohmic resistance of $10 \Omega \mathrm{cm}^{2}$ and a double-layer capacitance of $20 \mu \mathrm{F} / \mathrm{cm}^{2}$ for different values of $i / i_{\lim }$ : a) 0 ; b) $1 / 4$; d) $1 / 2$; e) $3 / 4$; and f) 1 . A line indicates the point on the polarization curve (c) corresponding to each Nyquist diagram. Characteristic frequencies are labeled.

After algebraic manipulation, the difference between Equations 48 and 49 may be expressed as

$$
\frac{\partial}{\partial t}\left(c_{\mathrm{AB}}-\frac{c_{\mathrm{B}^{+}}}{K_{\mathrm{eq}}}\right)=D \frac{\partial^{2}}{\partial y^{2}}\left(c_{\mathrm{AB}}-\frac{c_{\mathrm{B}^{+}}}{K_{\mathrm{eq}}}\right)-k\left(c_{\mathrm{AB}}-\frac{c_{\mathrm{B}^{+}}}{K_{\mathrm{eq}}}\right)
$$

where $k=k_{\mathrm{f}}+k_{\mathrm{b}}$ and $K_{\mathrm{eq}}=k_{\mathrm{f}} / k_{\mathrm{b}}$. As Equations 51 and 52 are linear, the solution for the convective-diffusion impedance does not require a solution for the steady-state. Equations 51 and 52 in the frequency domain were solved for the boundary conditions

$$
\widetilde{c}_{\mathrm{AB}}(\delta)=\widetilde{c}_{\mathrm{B}^{+}}(\delta)=0
$$




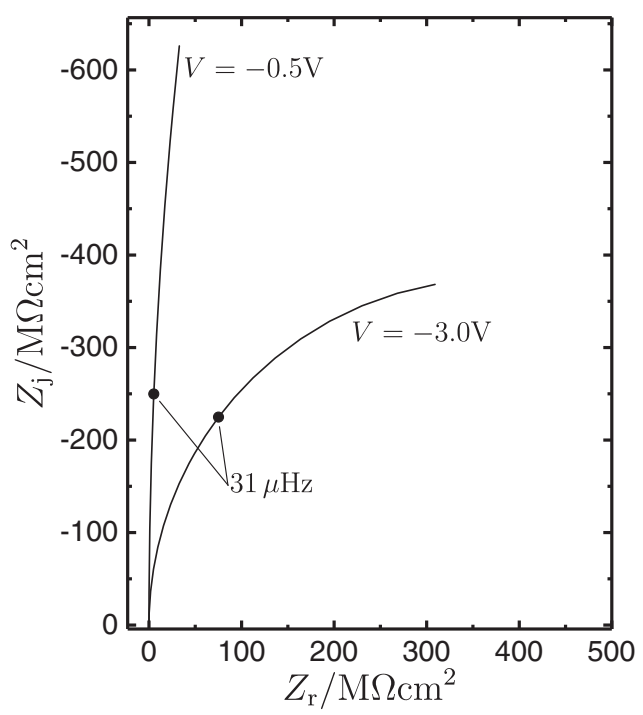

Figure 11. The overall impedance presented corresponding to Figures 10a and $10 \mathrm{f}$ with an expanded frequency range.

$$
\widetilde{c}_{\mathrm{B}^{+}}(0)=1
$$

and

$$
\left.\frac{\mathrm{d} \widetilde{c}_{\mathrm{AB}}}{\mathrm{d} y}\right|_{y=0}=0
$$

The dimensionless diffusion impedance may be expressed as

$$
-\frac{1}{\theta_{\mathrm{B}^{+}}^{\prime}}=-\frac{1}{\delta} \frac{\widetilde{c}_{\mathrm{B}^{+}}(0)}{\left.\frac{\widetilde{c}_{\mathrm{B}^{+}}}{\mathrm{d} y}\right|_{y=0}}
$$

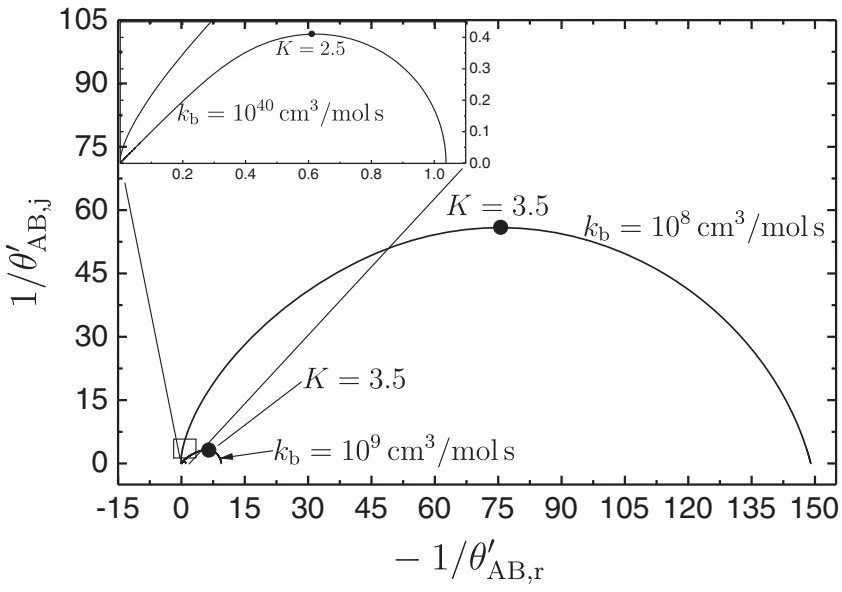

Figure 12. Dimensionless convective-diffusion impedance, defined by Equation 40, where the oscillating concentration of $\mathrm{AB}$ were taken into account in Equation 46 and large values of the homogeneous reaction rate constant were used.

$$
=\frac{1}{K_{\mathrm{eq}}+1} \frac{\tanh \sqrt{(\mathrm{j} \omega+k) \frac{\delta^{2}}{D}}}{\sqrt{(\mathrm{j} \omega+k) \frac{\delta^{2}}{D}}}+\frac{K_{\mathrm{eq}}}{K_{\mathrm{eq}}+1} \frac{\tanh \sqrt{\mathrm{j} \omega \frac{\delta^{2}}{D}}}{\sqrt{\mathrm{j} \omega \frac{\delta^{2}}{D}}}
$$

or

$$
-\frac{1}{\theta_{\mathrm{B}^{+}}^{\prime}}=\frac{1}{K_{\mathrm{eq}}+1} \frac{\tanh \sqrt{\mathrm{j} K+k_{\mathrm{dim}}}}{\sqrt{\mathrm{j} K+k_{\mathrm{dim}}}}+\frac{K_{\mathrm{eq}}}{K_{\mathrm{eq}}+1} \frac{\tanh \sqrt{\mathrm{j} K}}{\sqrt{\mathrm{j} K}}
$$

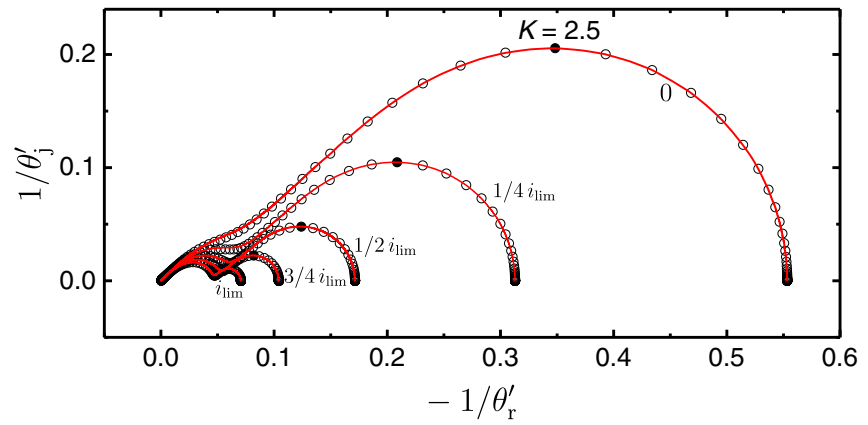

(a)

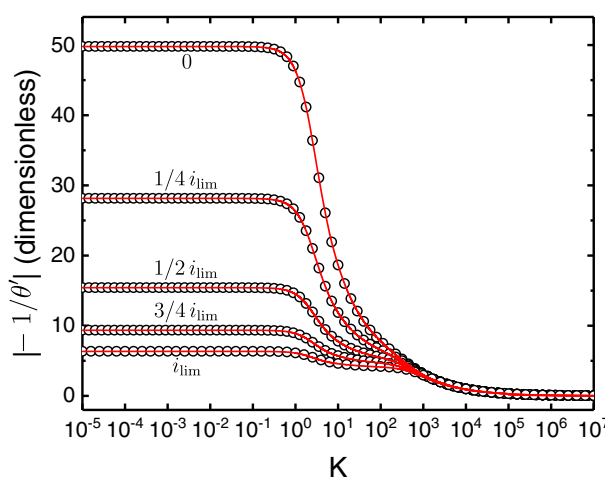

(b)

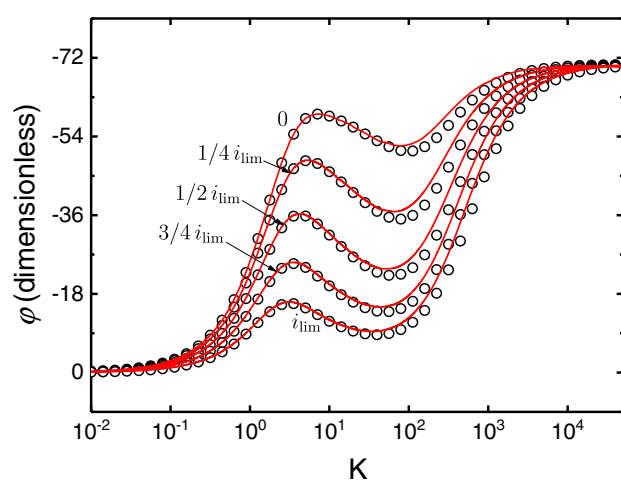

(c)

Figure 13. Comparison of simulated dimensionless convective-diffusion impedance, defined by Equation 40 and taken from Figure 7 for $k_{\mathrm{b}}=10^{7}$ at different fractions of the limiting current, to results obtained by fitting Equation 59 shown in a) Nyquist form, b) Bode Magnitude plot and c) Bode Phase plot. 
Table III. Fitting parameters found from regression using Equation 59 and Figure 13.

\begin{tabular}{|c|c|c|c|c|c|c|}
\hline Potential, V & $i / i_{\lim }$ & $k_{\mathrm{b}}, \mathrm{cm}^{3} / \mathrm{mol} \mathrm{s}$ & $k_{\mathrm{dim}}$ & $K_{\mathrm{eq}}$ & $\mathrm{Sc}$ & $\delta_{\mathrm{r}}, \mu \mathrm{m}$ \\
\hline-0.5 & 0 & $10^{7}$ & $197 \pm 5.5$ & $0.990 \pm 0.0057$ & $521 \pm 85$ & $0.98 \pm 0.014$ \\
\hline-1.65 & 0.05 & $10^{7}$ & $200 \pm 4.6$ & $0.789 \pm 0.0042$ & $528 \pm 87$ & $0.98 \pm 0.011$ \\
\hline-1.7549 & 0.25 & $10^{7}$ & $230 \pm 3.1$ & $0.340 \pm 0.0016$ & $528 \pm 101$ & $0.910 \pm 0.0062$ \\
\hline-1.8262 & 0.50 & $10^{7}$ & $301 \pm 3.0$ & $0.1309 \pm 0.00075$ & $502 \pm 134$ & $0.795 \pm 0.0039$ \\
\hline-1.85 & 0.59 & $10^{7}$ & $333 \pm 3.2$ & $0.0952 \pm 0.00062$ & $494 \pm 151$ & $0.756 \pm 0.0035$ \\
\hline-1.8938 & 0.75 & $10^{7}$ & $392 \pm 3.2$ & $0.0570 \pm 0.00047$ & $447 \pm 168$ & $0.697 \pm 0.0028$ \\
\hline-2.0 & 0.95 & $10^{7}$ & $474 \pm 3.5$ & $0.0300 \pm 0.00034$ & $386 \pm 78$ & $0.634 \pm 0.0023$ \\
\hline-3.0 & 1.0 & $10^{7}$ & $493 \pm 3.6$ & $0.0260 \pm 0.00032$ & $368 \pm 189$ & $0.621 \pm 0.0023$ \\
\hline-1.7674 & 0.50 & $10^{6}$ & $21.4 \pm 0.4$ & $0.353 \pm 0.0058$ & $249 \pm 78$ & $2.98 \pm 0.027$ \\
\hline-1.8951 & 0.50 & $10^{8}$ & $5240 \pm 39$ & $0.0355 \pm 0.00015$ & $652 \pm 178$ & $0.1906 \pm 0.001$ \\
\hline-1.9646 & 0.50 & $10^{9}$ & $98900 \pm 670$ & $0.0090 \pm 0.00037$ & $554 \pm 143$ & $0.0439 \pm 0.00015$ \\
\hline
\end{tabular}

Regression of Equation 58 to simulated diffusion impedances yielded unsatisfactory results. Thus, a modified Gerischer impedance was introduced in which the second term was replaced by an expression for the convective-diffusion impedance of a disk electrode under assumption of a finite Schmidt number, i.e.,

$$
-\frac{1}{\theta_{\mathrm{B}^{+}}^{\prime}}=\frac{1}{K_{\mathrm{eq}}+1} \frac{\tanh \sqrt{\mathrm{j} K+k_{\mathrm{dim}}}}{\sqrt{\mathrm{j} K+k_{\mathrm{dim}}}}+\frac{K_{\mathrm{eq}}}{K_{\mathrm{eq}}+1} \frac{-1}{\theta_{\mathrm{CD}}^{\prime}}
$$

where $-1 / \theta_{\mathrm{CD}}^{\prime}$ can be obtained using $K=\omega \delta_{\mathrm{B}^{+}}^{2} / D_{\mathrm{B}^{+}}$and a look up table generated from the solution of the convective-diffusion impedance without homogeneous reaction. ${ }^{39,40,42}$

Modified Gerischer impedance.-A Levenberg-Marquardt regression was used in Origin 2017 to regress Equation 59 to the data shown in Figure 7. The extracted parameters were $k_{\mathrm{dim}}, K_{\mathrm{eq}}$, and the Schmidt number. A comparison between the fit and the simulation results are shown in Figure 13 as Nyquist and Bode plots. Fitting parameters are listed in Table III.

The parameter $K_{\text {eq }}$ was found to vary with potential and rate constant $k_{\mathrm{b}}$, even though the equilibrium constant used for the simulations and reported in Table II was unchanged. This result is consistent with the observation that the assumptions employed for the Gerischer impedance are much more restrictive than those used for the numerical simulations. The regressed value of $K_{\text {eq }}$ serves to weight the homogeneous reaction and convective-diffusion loops as shown in Equation 59.

The Schmidt number obtained from regression had a large confidence interval which generally encompassed the input value of $\mathrm{Sc}=526$. The exception is seen for $k_{b}=10^{6} \mathrm{~cm}^{3} / \mathrm{mol} \mathrm{s}$, where the reaction layer thickness is on the order of $3 \mu \mathrm{m}$ (22 percent of the Nernst diffusion-layer thickness), extending into the region where the assumption of a Nernst stagnant diffusion layer is no longer ap- propriate for the homogeneous reaction zone. The tightest confidence intervals were found for situations for which the loop associated with the convective-diffusion impedance was much larger than that for the homogeneous reaction layer.

Following the definition of $k_{\mathrm{dim}}=k \delta^{2} / D$ and Equation 1, a relationship between the reaction thickness $\delta_{\mathrm{r}}$ and the Nernst diffusion layer thickness was found to be

$$
\delta_{\mathrm{r}}=\delta_{\mathrm{N}, \mathrm{B}^{+}} \sqrt{\frac{1}{k_{\mathrm{dim}}}}
$$

The reaction thicknesses obtained from Equation 60 are shown in Figure 14. The reaction distribution was scaled by the corresponding values at $y \rightarrow 0$ to emphasize the relative changes in values. As the heterogeneous reaction becomes bigger the reaction thickness decreases. Error bars are shown and were calculated using a linear propagation of errors from regressed values of $k_{\mathrm{dim}}$. This work demonstrates that a modified Gerischer impedance may be used to provide meaningful parameters for the general case for a rotating disk in which diffusion coefficients are not equal and the homogeneous reaction is not linear.

\section{Conclusions}

A mathematical model was developed for the impedance response associated with the coupled homogeneous chemical and heterogeneous electrochemical reactions. The general reaction of species $\mathrm{AB}$ reacting reversibly to form $\mathrm{A}^{-}$and $\mathrm{B}^{+}$and $\mathrm{B}^{+}$reacting electrochemically on a rotating disk electrode to produce $\mathrm{B}$ was studied. The resulting convective-diffusion impedance had two asymmetric capacitive loops, one associated with convective-diffusion impedance the other with the homogeneous reaction. The overall impedance can have

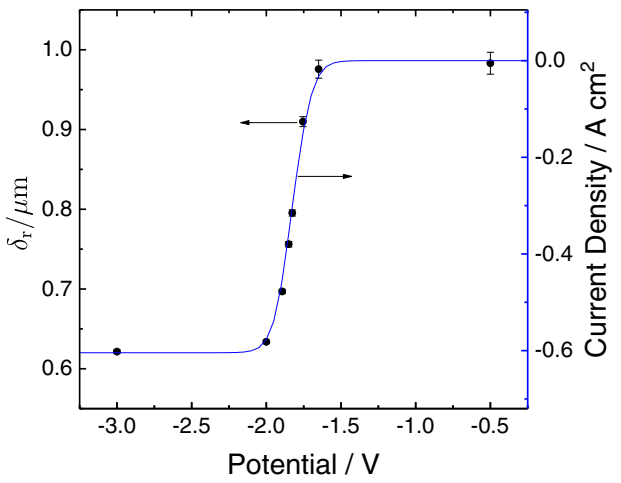

(a)

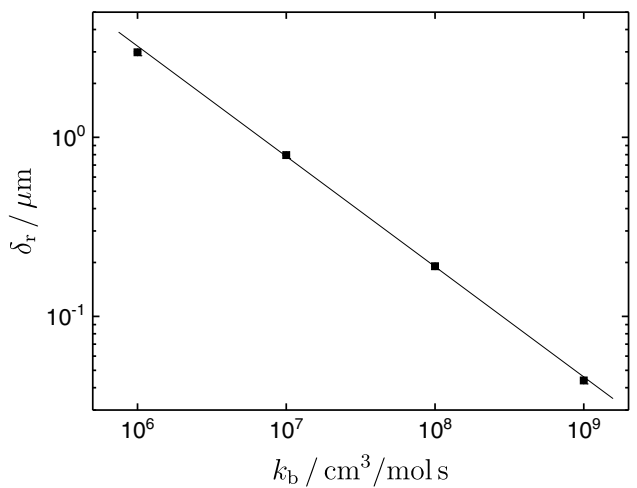

(b)

Figure 14. Reaction-layer thickness obtained from Equation 60 with regression parameters obtained by regressing Equation 59 to the simulated convectivediffusion impedance: a) reaction-layer thickness and current density as a function of potential and b) reaction-layer thickness as a function of homogeneous reaction rate. 
three time constants, associated with capacitance, homogeneous reaction and mass transport. For an infinitely fast homogeneous reaction, the system was shown to behave as though $\mathrm{AB}$ is the electroactive species and a convective-diffusion impedance was obtained. A modified Gerischer impedance was found to provide a good fit to the simulated data. The regression parameter $k_{\mathrm{dim}}$ was used to extract a reaction layer thickness. The modified Gerischer impedance provides an analytic approximation to the convective-diffusion impedance for a rotating disk in the presence of a chemical/electrochemical (CE) mechanism.

\section{Acknowledgment}

The authors gratefully acknowledge the financial support of their home institutions which facilitated this collaboration. The support of Medtronic Diabetes (Northridge, CA), Rui Kong and Andrea Varsavsky, program monitors, is gratefully acknowledged. Ambrose Douglas, an undergraduate researcher, helped create the code needed to perform the regression analysis.

\section{References}

1. J. A. Koutecky and V. G. Levich, Doklady Akademii Nauk, 117, 441 (1957).

2. J. A. Koutecky and V. G. Levich, Zhurnal Fizicheskoi Khimii, 32, 1565 (1958).

3. V. G. Levich, Physicochemical Hydrodynamics, Prentice Hall: Englewood Cliffs, 1962.

4. B. Bossche, L. Bortler, J. Deconinck, S. Vandeputte, and A. Hubin, Journal of Electroanalytical Chemistry, 397, 35 (1995).

5. T. von Kármán, Zeitschrift für angewandte Mathematik und Mechanik, 1, 233 (1921)

6. C. Deslouis, I. Grateur, G. Maurin, and B. Tribollet, Journal of Applied Electrochemistry, 27, 482 (1997).

7. E. Remita, B. Tribollet, E. Sutter, V. Vivier, F. Ropital, and J. Kittel, Corrosion Science, 50, 1433 (2008).

8. T. Tran, B. Brown, S. Nešiú, and B. Tribollet, Corrosion Science, 70, 223 (2014).

9. D. E. Smith, Analyrical Chemistry, 35, 602 (1963).

10. D. E. Smith, Analytical Chemistry, 35, 610 (1963).

11. R. Jurczakowski and P. Polczyǹski, Journal of Physical Chemistry C, 118, 7980 (2014).

12. P. Delahay and T. Berzins, Journal of the American Chemical Society, 75, 2486 (1953)
13. J. Savéant and E. Vianello, Electrochimica Acta, 8, 905 (1963).

14. R. S. Nicholson and I. Shain, Analytical Chemistry, 36, 706 (1964).

15. H. Gerischer, Zeitschrift für physikalische Chemie (Neue Folge), 197, 92 (1951)

16. A. Lasia, Electrochemical impedance spectroscopy and its applications; Springer, 2014.

17. V. V. Pototskayaa and O. I. Gichan, Electrochimica Acta, 235, 583 (2017).

18. W. Thomas and R. A. Chapman, Electochimica Acta, 56, 128 (2010).

19. E. Levart and D. Schuhmann, Journal of Electroanalytical Chemistry, 53, 77 (1974).

20. B. Tribollet and J. S. Newman, Journal of the Electrochemical Society, 131, 2780 (1984).

21. A. K. Hauser and J. S. Newman, Journal of the Electrochemical Society, 136, 2896 (1989).

22. A. K. Hauser and J. S. Newman, Journal of the Electrochemical Society, 136, 3319 (1989).

23. J. Vazquez-Arenas and M. Pritzker, Electrochimica Acta, 55, 8376 (2010).

24. J. Vazquez-Arenas and M. Pritzker, Electrochimica Acta, 56, 8023 (2011).

25. S. B. Adler, Chemical Reviews, 104, 4791 (2004)

26. S. B. Adler, J. A. Lane, and B. C. H. Steele, Journal of The Electrochemical Society, 143, 3554 (1996).

27. S. B. Adler, Solid State Ionics, 111, 125 (1998).

28. R. Schiller, J. Balog, and G. Nagy, The Journal of Chemical Physics, 123 (2005).

29. A. B. Boukamp and H. Bouwmeester, Solid State Ionics, 157, 29 (2003).

30. R. Kumar and R. Kant, The Journal of Physical Chemistry C, 113, 19558 (2009).

31. A. Jukic and M. Metikoš-Hukovic, Electrochimica Acta, 48, 3929 (2003); Electrocatalysis:From Theory to Industrial Applications.

32. N. Anicet, C. Bourdillon, C. Demaille, J. Moiroux, and J.-M. Savant, Journal of Electroanalytical Chemistry, 410, 199 (1996).

33. S. Updike and G. Hicks, Nature, 214, 986 (1967)

34. A. Heller and B. Feldman, Chemical Reviews, 108, 2482 (2008).

35. J. S. Newman and K. E. Thomas-Alyea, Electrochemical Systems, 3rd ed.; John Wiley \& Sons: Hoboken, 2004.

36. W. G. Cochran, Proceedings of the Cambridge Philosophical Society, 30, 365 (1934).

37. R. E. White, C. M. Mohr Jr., and J. S. Newman, Journal of the Electrochemical Society, 123, 383 (1976).

38. S.-L. Wu, Ph.D. thesis; University of Florida; Gainesville, FL; 2010.

39. M. E. Orazem and B. Tribollet, Electrochemical Impedance Spectroscopy; John Wiley \& Sons: Hoboken, 2008.

40. M. E. Orazem and B. Tribollet, Electrochemical Impedance Spectroscopy, 2nd ed.; John Wiley \& Sons: Hoboken, 2017.

41. M. T. T. Tran, B. Tribollet, V. Vivier, and M. E. Orazem, Russian Journal of Electrochemistry, in press (2017).

42. B. Tribollet and J. S. Newman, Journal of the Electrochemical Society, 130, 822 (1983). 